\title{
Gray Box Modelling of Arterial Vasoaction
}

\author{
†Violeta Mangourova and *John Ringwood \\ Department of Electronic Engineering \\ National University of Ireland, Maynooth \\ IRELAND \\ E-mail: †violeta.i.mangourova@nuim.ie ${ }^{*}$ john.ringwood@eng.nuim.ie
}

\begin{abstract}
Gray box modelling of physiological systems involves constructing a model structure based on physical knowledge of the system and model parameterisation using numerical techniques. This paper presents a gray box model of arterial vasoaction (the process of constricting and dilating blood vessels in order to maintain an appropriate level of blood pressure and blood flow). The model structure is built in accordance with the physical system . The initial parameterisation was manual, with the model consequently optimised using gradient techniques and genetic algorithms. The model was validated by demonstrating good correlation between experimental results and model output.

Keywords - gray box modelling, physiological systems, blood

flow, vasodilation
\end{abstract}

\section{INTRODUCTION}

Gray box modelling is a popular approach for modelling real systems, combining both black box and white box methodologies [1]. Black box modelling is a method, which uses no knowledge of the physical system, but is based on experimental data only. The exact opposite is white box modelling, where all necessary information about the system is available, therefore the model can be constructed directly from prior knowledge. Gray box modelling has the advantage of using both physical a priori knowledge of the system for model structure development, and parameter estimation from available experimental data.

Gray box modelling is an increasingly popular approach for studying physiological systems. In most such cases, the input/output relationship of a system is not of paramount importance, as the aim of the model is to develop a greater understanding of the physical system and its underlying processes.

An important physiological process, which is the focus of numerous biomedical and bioengineering studies, is the regulation of blood pressure (BP). This process is critical in maintaining nutrient and oxygen supply to the various perfused organs, hence an accurate model is necessary in order to achieve a true understanding of the system. Multiple interconnecting mechanisms are involved in blood flow and blood pressure regulation, posing a difficulty in developing a simple mathematical model from first principles.
This present study will attempt to model short term blood flow/pressure regulation, while assuming that the primary pump, the heart, has a relatively constant mean output. Familiarity with the processes can help outline the model structure, while the parameters can be tuned, based on available experimental data. However, if the underlying model structure is poor, it is unlikely that even the most powerful optimisation techniques can achieve an acceptable data fit. Manual, deterministic and probabilistic search approaches were employed.

Deterministic optimisation is an approach that yields identical solutions when the same initial conditions are defined, for example Gradient descent, Newton and Gauss-Newton. They are popular due to their simplicity and the fact that, at least, a local minimum solution is almost guaranteed. The algorithm used in this study is the quasi-Newton method, the rational for which is explained in Section III (b).

Stochastic optimization uses a degree of randomness in its search methodology and gives different solutions even when the same starting point is used, which can help to combat premature convergence due to local minima. Some examples of stochastic algorithms are genetic algorithms (GAs) and simulated annealing. In this study, genetic algorithms have been used extensively. They are useful for optimization of problems with irregular solution surfaces, as they can explore a large solution space with multiple trials.

The remainder of the paper is organised as follows: Sections II and III deal with the model 
structure determination and optimisation techniques used, while available experimental data and model results are presented in Sections IV and $\mathrm{V}$ respectively. The conclusions are laid out in Section VI.

\section{PHYSIOLOGICAL MODEL FOR VASOACTION}

This study will focus on modelling short term renal (kidney) blood flow regulation. A basic blood pressure equation can be expressed in the form of Ohm's Law as follows:

$$
M A P=C O . T P R
$$

where:

MAP is the mean (of the systolic and diastolic) pressure (measured in $\mathrm{mmHg}$ ),

CO is cardiac output, evaluated as the product of heart rate and stroke volume (in $\mathrm{l} / \mathrm{s}$ ), and

TPR is the total peripheral resistance as seen by the heart (in $\mathrm{mmHg} \mathrm{s/l}$ ).

As $\mathrm{CO}$ is taken to be constant, the main control parameter in this case will be the peripheral resistance. This assumption is acceptable as the heart rate variability during SNA stimulation was shown to be very small (e.g. $\mathrm{HR}=233.59 \pm 2$ beats per min. for frequency of stimulation of $5 \mathrm{~Hz}$ ).

A number of factors, both systemic (hormonal, neural) and local (tissue metabolites and paracrine), are involved in blood flow regulation. The change in the levels of these important factors can result in adjustment of the TPR, hence causing vasoconstriction or vasodilation.

Central to the short timescale of interest to us, with a time delay between stimulation and response of approximately $0.6 \mathrm{~s}$ [2], is the neural control of blood pressure through sympathetic nerve activity (SNA). However, in addition to neural control, several other mechanisms have significant effect on resistance, including:

- Hormones, which can effect both vasodilation or vasoconstriction, depending on the particular hormone and the type of receptor it binds to ( e.g. Epinephrine, Antidiuretic Hormone, Angiotensin II and Cortisol) [3, 4],

- Intrinsic factors (myogenic autoregulation), which regulate blood vessel compliance in response to a distorting force on the walls due to blood pressure [5],

- Paracrines, which are humoral substances that are secreted by cells in the endothelium, and have vasodilatory effects(e.g. Prostacyclin, Nitric Oxide), vasoconstrictory effects (e.g. Thromboxane, Endothelin-1), or both (e.g. Endothelin-1 [6]).

- Metabolic factors, which can elicit vasoaction in response to local metabolic demands. Typical mediators include oxygen (constriction).

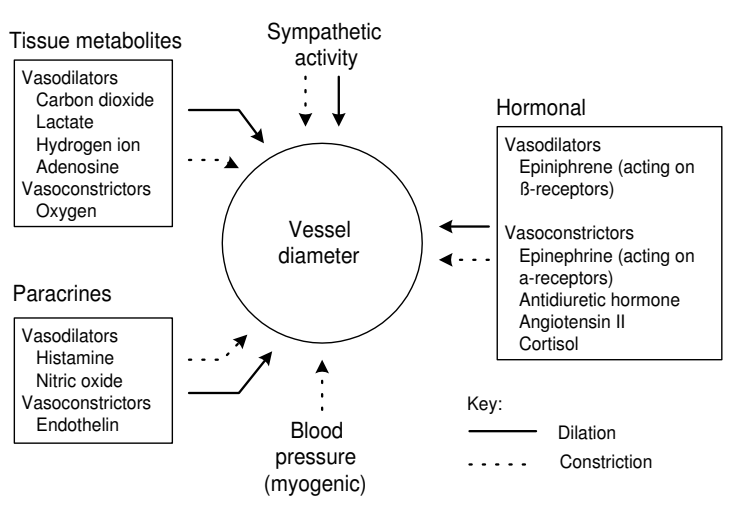

Fig. 1: Summary of vasoactive mechanisms

Fig. 1 (adapted from [7]) attempts to summarise the various factors involved in mediating vasoaction. These factors, and their activation mechanisms, will be used to form the basic structure of the model. The SNA input will be the only openloop component in the model. This open-loop representation is due to the fact that the experimental procedure included severing of the renal sympathetic nerves and application of artificial SNA simulation. A simple representation of the structure of the gray box model is shown in Fig. 2.

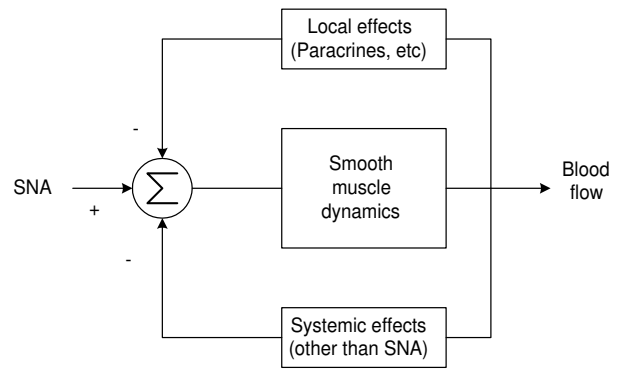

Fig. 2: Feedforward/feedback configuration

The feedforward path represents the neural mechanism for renal blood flow regulation. The two feedback paths represent the effects that both local and systemic blood pressure/flow control mechanisms have in response to SNA-based activation of renal blood flow.

To date, a number of attempts have been made to model the blood flow response to SNA. However, most focus on black-box techniques, which have no physical meaning and also fail to capture essential aspects of the response [2, 8, 9]. 


\section{PARAMETER OPTIMISATION}

Various optimization techniques can be used to parameterise the gray box model and the objective function to be minimised is defined as:

$$
J=\frac{1}{N} \sum_{i=1}^{N}\left(y_{i}-\hat{y}_{i}\right)^{2}
$$

where,

- $\hat{y}_{i}$ is the blood flow data obtained from the model simulations, and

- $y_{i}$ is the original blood flow data

The model was tested for frequencies of simulation of $1.5,2.0,3.0,5.0$ and $8.0 \mathrm{~Hz}$ and the parameters were determined to give optimum results for all frequencies of simulation.

\section{a) Manual Tuning}

Given the intuitive nature of the model and the strong relationship with the underlying physiology, initial attempts focussed on tuning the model parameters by trial and error. There were 4 parameters to determine and two piecewise linear functions. The relationship between SNA and blood flow is approximately linear above a certain threshold level of renal blood flow, hence the feedforward loop dynamics are relatively easy to predict. However, below that threshold level, local factors are activated to oppose the reduction in blood flow, thus activating the feedback path in the model. This latter relationship is nonlinear, hence more challenging to parameterise 'by hand'. However, there is a reasonably strong relationship between the parameters and blood flow response, hence the manual tuning was justified as a starting point of the model parameterisation.

\section{b) Gradient Search}

The first attempt at numerical optimisation in this study involved gradient search techniques. A quasi-Newton algorithm was employed to determine the global minimum of the objective function. Most Newton gradient search algorithms require calculations of the gradient and Hessian (second partial derivative, representing the curvature). Numerical calculation of the Hessian is usually computed by dividing a difference by a very small quantity, which can lead to loss of precision and even divergence from the minimum, in some cases. However, in the quasi-Newton method, the parameter approximations for the next step are calculated using estimates of the Hessian, calculated in a specific manner to reduce precision loss [10].

\section{c) Stochastic Methods}

Stochastic methods are probabilistic search methods and, when combined with concurrent methods, they support a set of individuals containing information for each unknown parameter. These methods offer a set of solution vectors at every iteration and the fittest individuals can proliferate to improve the solution set in future generations.

GAs, which are members of the family of concurrent and stochastic search methods, attempt to mimic the natural selection process by a number of specific operators: selection, mutation and crossover. At every generation (iteration), the fitness of each individual is assessed and the fittest members of the population are selected for the recombination process. These fittest individuals recombine and then mutate in order to produce a new generation of solutions. However, the effectiveness of the algorithm can depend on the encoding technique used. GAs can be coded either as binary strings, real numbers, integers etc.

\section{DATA AVAILABILITY}

Experiments were performed on 6 anaesthetized New Zealand white rabbits at the Circulatory Control Lab., University of Auckland [11]. A transit time flow probe (type 2SB; Transonic Systems, Ithaca, NY, USA), connected to a compatible flowmeter (T106, Transonic Systems) was used to measure renal blood flow (RBF), with arterial pressure being monitored using a catheter inserted into the central ear artery and connected to a pressure transducer (Cobe, Arvarda, CO). The measured signals were sampled at $500 \mathrm{~Hz}$, digitized and saved continuously as $2 \mathrm{~s}$ averages of each variable. In addition, heart rate (HR, beats/min) was derived from the MAP waveform.

For stimulation, the renal nerves were placed across a pair of hooked stimulating electrodes and then sectioned proximal to the electrodes. Stimulation sequences using both amplitude (AM) and frequency modulation (FM) were applied, all using a pulse width of $2 \mathrm{~ms}$. In the AM sequence, voltages of $0.5,1.0,1.5,2.0,3.0,5.0$ and $8.0 \mathrm{~V}$ were applied in random order at a constant frequency of $5 \mathrm{~Hz}$. For the FM sequence, frequencies of 0.5 , $1.0,1.5,2.0,3.0,5.0$ and $8.0 \mathrm{~Hz}$ were applied in random order using a voltage equal to that required to produce a maximal RBF response. For both AM and FM sequences, the stimulation interval was 3 min., with a 5 min. recovery period before delivering the next stimulus. 


\section{MODELLING RESULTS}

\section{a) Model Structure}

Since the renal vasculature is just one component which regulates blood pressure, the response from systemic mechanisms is unlikely to be nearly as significant as the response from local mechanisms. Therefore, a single feedback block will be employed in the structure as shown in the block diagram of Fig.3.

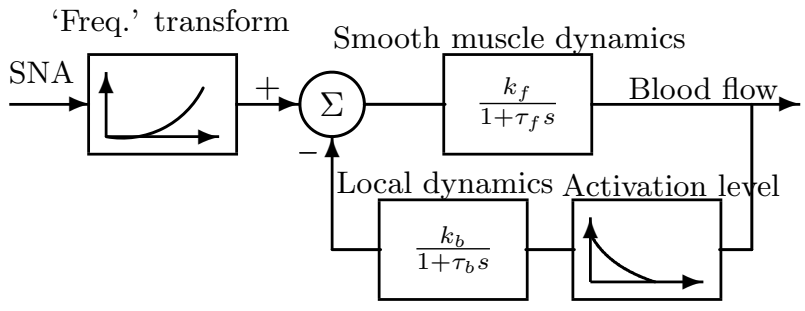

Fig. 3: Block diagram of proposed model structure

One further component was added to the basic structure shown in Fig.2, in order to correctly represent the relationship between the varying levels of steady state blood flow response to the frequency of SNA stimulation. This (mildly) nonlinear characteristic is represented in Fig.3 by the 'Freq. transform' block.

The model structure is based on the following physiological premise. Above a certain (threshold) value of normal blood flow, the response of blood flow to SNA is relatively linear. The 'Smooth muscle dynamics' block in Fig.3 represents this dynamic response of the vascular smooth muscle to stimuli. The effect of SNA is that of vasoconstriction and the speed and magnitude of the response are defined by the corresponding pole location (time constant) and gain respectively. However, when blood flow drops below a certain value, local myogenic factors and paracrines work progressively harder (as blood flow decreases) to maintain an acceptable level of local blood flow, causing reactive vasodilation. This combination of threshold and progressive response is captured by the 'Activation level' block in Fig.3, while the 'Local dynamics' block captures the speed of response of these local reactionary mechanisms.

Fig. 4 shows the typical response shape obtained from the model. The initial response to a step activation in SNA is roughly first order exponential, but as soon as blood flow reduction reaches a certain level, local (opposite) effects temper the response dramatically. Following release of the SNA activation, the response returns rapidly to the original level, assisted by the local paracrines, etc. which are still active and have not yet been dispersed. Finally, the blood flow overshoots its

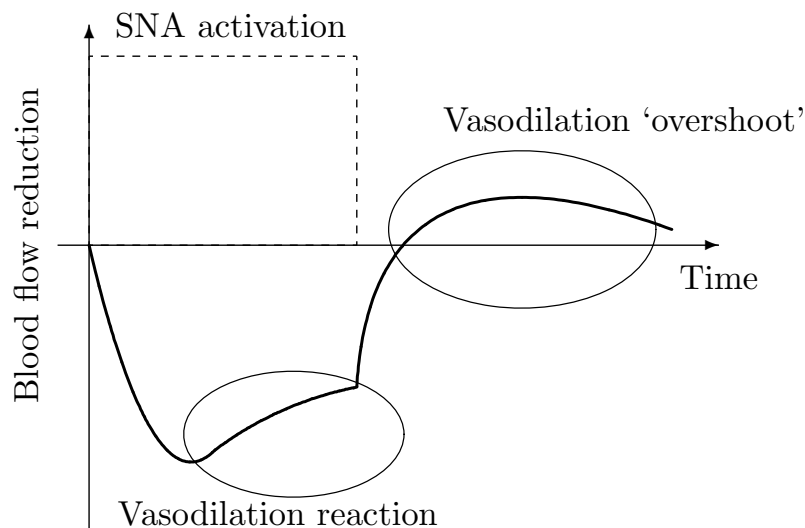

Fig. 4: Typical 'large-signal' response to SNA activation

original value, due to the slow dispersal of these local vasodilatory effects.

From the above description, some aspects of the model can be clarified:

- The local vasodilatory response is not linear and has some 'threshold' of blood flow change above which it is activated,

- The response of the local vasodilatory reaction is significantly slower than that of the smooth muscle to the SNA stimulus (i.e. $\tau_{b}>\tau_{f}$ ), and

- The magnitude of the action (to SNA) and reaction (by the local vasodilatory mechanism) is comparable, at least to an order of magnitude.

\section{b) Parameter optimisation}

The parameters of the dynamic feedforward and feedback blocks, as well as the two nonlinear characteristics, were first determined by trial and error, then optimised using gradient techniques and genetic algorithms.

The model parameter values and minimum cost function obtained by the initial manual optimisation process are detailed in Tables 1 and 2 respectively.

Table 1: Dynamic blocks parameters for each of the three optimisation techniques - Manual, Gradient and GA

\begin{tabular}{|c|c|c|c|c|}
\hline & $k_{f}$ & $k_{b}$ & $\tau_{f}(\mathrm{~s})$ & $\tau_{b}(\mathrm{~s})$ \\
\hline Manual & 1 & 80 & 20 & 200 \\
\hline Gradient & 0.94 & 66.63 & 19.65 & 206.87 \\
\hline GA & 1.05 & 81.58 & 20.37 & 203.85 \\
\hline GA+ Grad & 1.04 & 72.15 & 20.09 & 179.98 \\
\hline
\end{tabular}

A demonstration of the nonlinear feedback 'activation level' function input/output relationship is shown in Fig 5 . 
Table 2: Minimum cost functions for each of the three optimisation techniques - Manual, Gradient and GA

\begin{tabular}{|c|c|}
\hline OptimisationTechnique & MinimumCost \\
\hline Manual & 234.59 \\
\hline Gradient & 109.19 \\
\hline GA & 107.82 \\
\hline GA + Grad & 102.46 \\
\hline
\end{tabular}

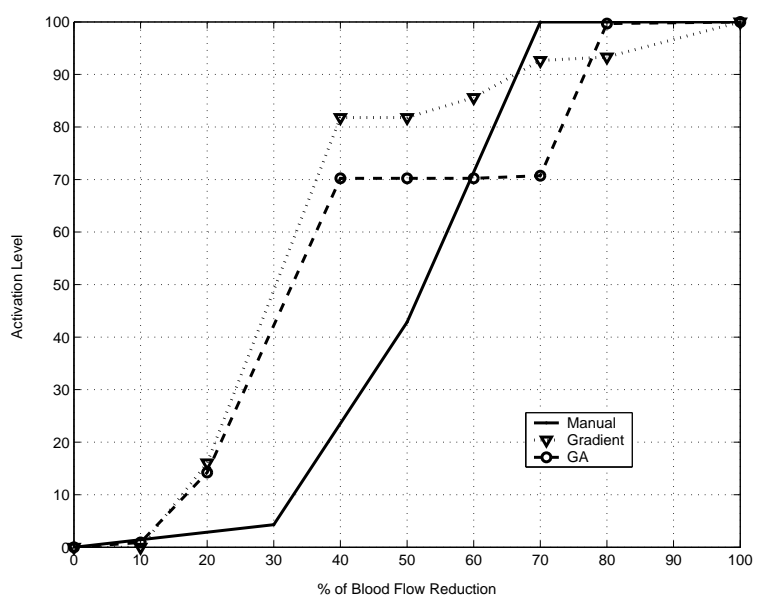

Fig. 5: Feedback activation function

Furthermore, the nonlinear function, representing the steady-state relationship between frequency of SNA and blood flow response, is shown in Fig. 6.

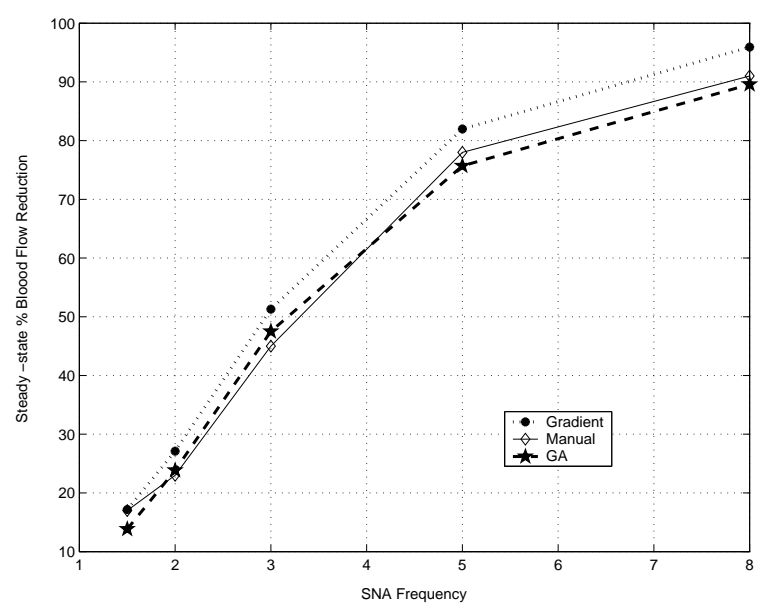

Fig. 6: Transformation for frequency stimulation

The performance of the model in comparison to the recorded experimental data may now be evaluated via Fig.7.

Clearly, even with manual tuning, the model has captured the essence of the response contained in the the experimental data, with the exception of the noise present in the physiological data. However, it is not the intention of the model to represent this noise. Arguably a better comparison could be made if the experimental data had been filtered prior to plotting, but the filter would in-

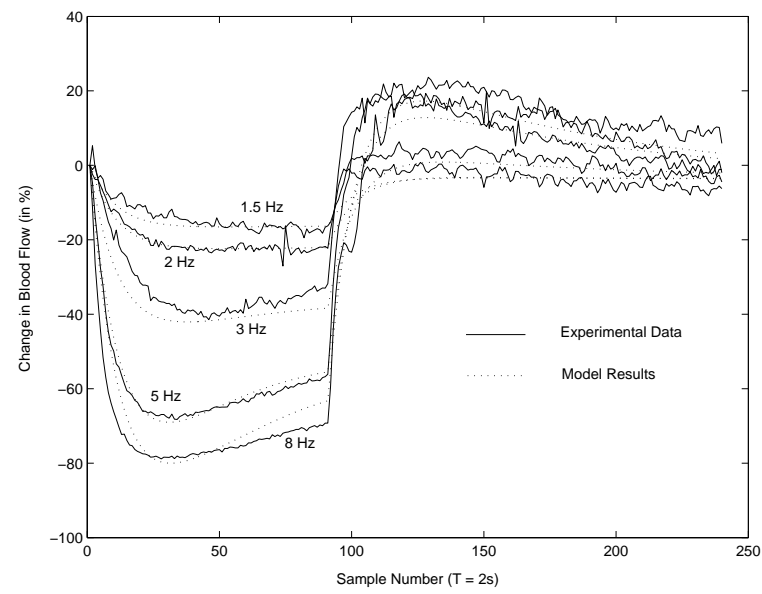

Fig. 7: Comparison of model response with experimental data (Manually optimised parameters)

troduce dynamics of its own, which may interfere with the parameter determination.

Following the manual tuning of the model, we applied a gradient algorithm to fine-tune the parameters. In addition, the representation of the local vasodilator 'Activation level block' was extended to increase its flexibility. Numerous simulations of the model were completed and we noted that the performance of the search algorithm was highly dependent on the initial conditions, thus concluding that the performance surface is highly irregular.

The minimum value for the cost function obtained with the quasi-Newton method and the corresponding parameter values are shown in Table 1 and Table 2 respectively, which represent a modest improvement over the manual tuning case. However, due to the irregularity of the surface, we concluded that the optimum solution will not be reached with a gradient optimisation technique, therefore GAs were applied to the problem. A binary encoding technique was selected initially, but as the algorithm evolved, real numbers were used, because the problem was not binary in nature. Large numbers of individuals (more than 100) and up to 100 iterations were used during each search to ensure that a wide solution space was covered. The obtained parameter values using GAs for the feedback and feedforward blocks, and the corresponding minimum cost function, are also displayed in Tables 1 and 2 .

A further enhancement of the parameter values can be achieved by using the end point of the GA solution as a starting point for a gradient technique to fine-tune the parameter values. The results are again included in Tables 1 and 2 and indicate a modest improvement in the cost. The model output, resulting from this last set of parameter values, is compared to the experimental data in Fig. 8, which shows an improvement over Fig.7. 


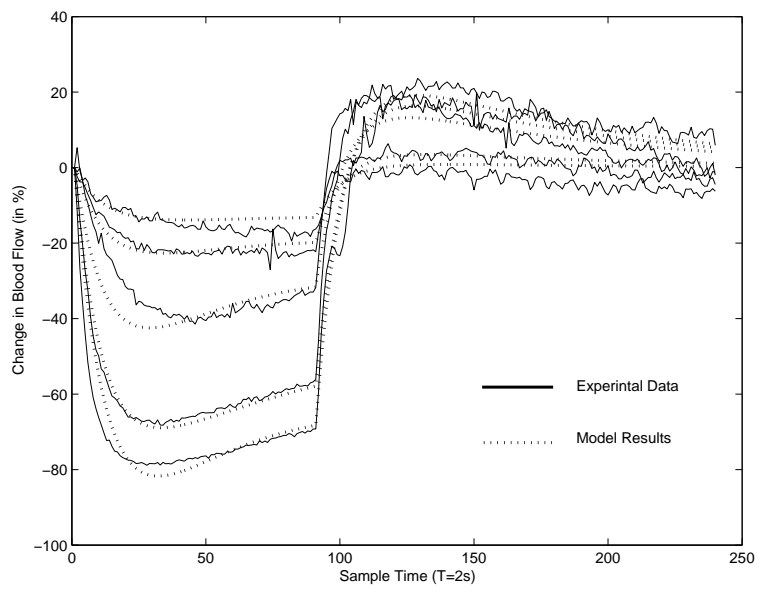

Fig. 8: Comparison of model response with experimental data (GA + gradient optimised parameters)

However, the relatively good fit for manual tuning gives credence to the chosen structure and indicates the physical transparency of the model.

\section{CONCLUSION}

A novel nonlinear gray box model for arterial vasoaction was developed in this study. It is based on underlying physiological principles and optimised using numerical methods, with best results given by a combination of genetic algorithms and gradient techniques. The model fit obtained was significantly better than previously reported models [11]. The model was validated by demonstrating a very good correlation between model results and experimental data, though a black box model could have possibly yielded a much better input/outpt match. This confirms the model structure employed, indicating the dynamics and activation levels associated with individual physiological components, which would remain invisible in a black box model. For example, the 'feedback activation function' shows the blood flow reduction levels at which the local vasodilatory effects become active (Fig.5).

Further enhancements will extend aspects of the model structure. Currently, the model does not include features to account for variability across different animals due, for example to different resting conditions (mean HR, MAP etc), hence some elements will be incorporated to achieve this improvement. In addition, a feature will be included in future models to take into account the different rates of responses in positive-going and negativegoing directions, clearly seen in Fig. 7, which are due to different activation and dispersion rates.

Finally, it is also anticipated that a model can be built to combine the effects of both amplitude and frequency-coded SNA stimulation and genetic programming can be employed to obtain an optimum structure of the model.

\section{ACKNOWLEDGEMENTS}

The authors are grateful for access to the experimental data used in this work and insightful comments provided by Prof. Simon Malpas and Dr. Sarah-Jane Guild of the University of Auckland.

\section{REFERENCES}

[1] K. Tan, Y. Li, P. Gawthrop, and A. Glidle, Evolutionary Grey-Box Modelling for Practical Systems. Technical Report CSC-96019, Centre for Systems and Control, University of Glasgow, 1997.

[2] S. Guild, P. Austin, M. Navatikyan, J. Ringwood, and S. Malpas, "Dynamic relationship between sympathetic nerve activity and renal blood flow: a frequency domain approach," Am. J. Physiol. (Regulatory Integrative Comp. Physiol.), vol. 281, pp. R206-R212, 2001.

[3] R. Bellomo, J. Kellum, S. Wisniewski, and M. Pinsky, "Effects of norepinephrine on the renal vasculature in normal and endotoxemic dogs," Am. J. Respir. Crit. Care Med., vol. 159, pp. R1186-R1192, 1999.

[4] C. Sorensen, P. Leyssac, O. Skott, and N.-H. Holstein-Rathlou, "Role of the renin-angiotensin system in regulation and autoregulation of renal blood flow," Am. J. Physiol. (Regulatory Integrative Comp. Physiol. ), vol. 279, pp. R1017-R1024, 2000.

[5] L. G. Navar, "Integrating multiple paracrine regulators of rrenal microvascular dynamics," Am. J. Physiol. (Renal Physiol.), vol. 274, pp. F433F444, 1998.

[6] C. Boulanger and P. Vanhoutte, "The endothelium: a modulator of cardiovascular health and disease," Dialogues in Cardiovascular Medicine, vol. 3, p. 4, 1998.

[7] D. Richardson, D. Randall, and D. Speck, Cardiopulmonary System, 1st ed. Fence Creek Publishing, 1998.

[8] G. Eppel, K. Denton, S. Malpas, and R. Evans, "Nitric oxide in responses of regional kidney perfusion to renal nerve stimulation and renal ischaemia," Eur. J. Physiol, vol. 447, pp. 205-213, 2003.

[9] M. Navakatikyan, B. Leonard, R. Evans, and S. Malpas, "Modeling the neural control of intrarenal blood flow," Clinical and Experimental Pharmacology and Physiology, vol. 27, pp. 650$652,2000$.

[10] R. Schoenberg, Optimization with the QuasiNewton Method. Aptech Systems, Inc. Maple Valley, WA, 2001.

[11] B. Leonard, R. Evans, M. Navakatikyan, and S. Malpas, "Differental neural cotrol of intrarenal blood flow," Am. J. Physiol. (Regul Ingr. Comp. Physiol), vol. 297, pp. R907-R916, 2000. 